\title{
STABILITY OF IONS IN THE ELECTRON BEAM WITH A GAP *
}

\author{
$\underline{\text { E. Bulyak }}^{\dagger}$, NSC KIPT, Kharkov, Ukraine
}

\section{Abstract}

A gap in the bunch train is one of methods to remove ions from the beam circulating in electron storage rings. Selfconsistent theory of the ion stability in the bunch train with a gap is developed out. As is shown, stability of ions increases with the increase in the ion core density. Under certain conditions that have been determined, the stability islands become overlapping. Therefore, the beam clearing may not be achieved. It is proved that the maximum charge neutralization of the circulating current reaches in the continuous beams, any gap decreases stable density of ions. The stable density of the ion core decreases with number of the ion oscillation within the bunch train increases.

\section{INTRODUCTION}

Positive ions produced by an electron beam from the residual gas being confined within the beam, can demolish the beam performance. For the most electron storage rings, these ions have to be extracted from the beam. A gap in the bunch train is one of methods to remove ions from the beam circulating in electron storage rings. The essence of this method is as follows. Injection of a beam is performed in a way that leaves a number of consecutive RF buckets empty. Thus, the ions experience periodic attractive (the force of partially neutralized electron macrobunch) and repulsive (the self force of the ion core) forces. Length of the empty part of a beam is chosen to provide unstable ion motion within the operating intensity of a beam. The stability of a single ion (the ion core of zero density) was estimated in $[1,2]$. As it was shown, the stability of an ion in the beam of a definite geometry is determined by the ion mass and the beam density. A real beam could confine the dense ion core before reaching the unstable conditions. The beam could trap the ions during the beam injection (when the beam density is below the unstable limit) or in operating cycle (when due to losses the beam enters the stable region from the above). Thus, during operation, the beam usually crosses the regions of ion stability. Therefore, of much importance is to establish the stable region border in the plane of beam density vs. ion core density.

\footnotetext{
* Work supported by the STCU, Project 855

† Email: bulyak@kipt.kharkov.ua
}

\section{MODEL}

Let us consider the ions moving within the beam, as these only ions affecting the beam particles. This assumption allows to simplify the task with least losses in generality. The following model is chosen (see Fig 1). The train of bunches (macrobunch) of the radius $a$, length $L_{t}$, and the longitudinal electron density $N_{t}$ are filled with ions of density $N_{i}$ (the train neutralization factor is $\eta_{t} \equiv N_{i} / N_{t}$ ).

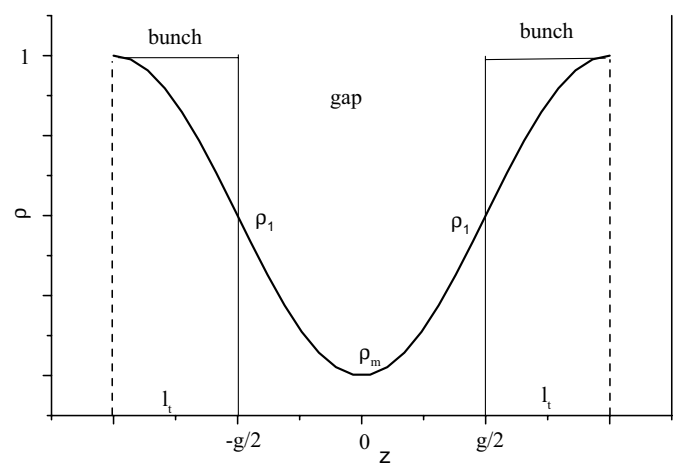

Figure 1: The model

We mean the bunch being long if an ion radius is changed significantly while the bunch passes by. The density limit of ion core provides that the clearing gap $G(\eta)$ between consecutive macrobunches causes the ion motion unstable.

From the physical point of view it follows that the phase volume of ion core decreases when the density is risen, therefore the core at the edge of equilibrium possess zero phase volume. The ions in this case move similarly (laminary). So, we can consider just dynamics of the boundary ions that are remaining in the border if the core is at the instability threshold.

The electrical field strength of the uniformly charged round rod of radius $r$ in its surface is:

$$
E_{r}\left(r^{\prime}=r\right)=\frac{N_{\mathrm{i}} e}{2 \pi \epsilon_{0} r} .
$$

The corresponding potential function reads as

$$
U_{i}(r)=-\frac{N_{i} e^{2}}{2 \pi \epsilon_{0}} \ln \frac{r}{a}=-2 N_{\mathrm{i}} R_{0} M c^{2} \ln \frac{r}{a},
$$


where $a$ is the initial core radius, $M$ is the ion mass; $R_{0}$ is the classical ion radius.

An attractive force acting on ions from the bunch space charge should be added to the repulsive force (1) of the core self-charge. Its potential is:

$$
U_{b}=N_{b} R_{0} M c^{2}\left(\frac{r}{a}\right)^{2} .
$$

Thus, we can write a (nonrelativistic) Hamilton function for the ion core border motion

$$
\begin{aligned}
H & =\frac{p^{2}}{2 M}+U(x) \\
& =\frac{p^{2}}{2 M}+R_{0} M c^{2}\left[\theta N_{b}\left(\frac{r}{a}\right)^{2}-N_{i} \ln \left(\frac{r}{a}\right)^{2}\right]
\end{aligned}
$$

Here we denote $\theta=1$ within the bunch, and $\theta=0$ outside (in the gap between consecutive bunches).

As we consider the bunch being long, the ion core can undergo a few oscillation while the bunch is passing by. The pulsation period can be derived from (4) put $p(r=$ $a)=0$. The hamiltonian (4) becomes:

$$
H=E=\text { const }=N_{b} R_{0} M c^{2} .
$$

At the loser crossover, $p\left(r=r_{m}\right)=0$, we have

$$
\rho^{2}-\eta_{t} \ln \rho^{2}=1
$$

where $\rho \equiv r / a$.

Equ. (6) has the root at $\rho=1$, the second root $\rho_{*}<1$ exists if the neutralization factor does not exceed unity: $\eta_{t}<1$. Taking this into account, from (4) we get an expression for the period $T$ of the border ion oscillation within the bunch:

$$
T_{i}=\frac{a}{c} \sqrt{\frac{2}{N_{i} R_{0}}} \int_{\rho *}^{1} \frac{d \rho}{\sqrt{1-\rho^{2}+\eta \ln \rho^{2}}} .
$$

As $\rho_{m}=\rho_{m}(\eta)$ (see (6)), we can denote

$$
\Psi(\eta) \equiv \int_{\rho_{m}(\eta)}^{1} \frac{d \rho}{\sqrt{1-\rho^{2}+\eta \ln \rho^{2}}},
$$

The pulsation length $\lambda=c T_{i}$ is dependent on the bunch radius and density, as well as on the ion mass and the neutralization factor

Due to symmetry or the considered system, the core envelope reaches its minimal crossover $\rho_{m}\left(\rho_{m} \geq \rho_{*}\right)$ just in the middle of the clearing gap between bunches (see Fig 1).

The trajectory of the border ions is described implicitly as

$$
\begin{aligned}
z & =z_{0}+\frac{a}{\sqrt{2 N_{e} R_{i}}} \int_{\rho_{1}}^{1} \frac{\mathrm{d} \rho}{\sqrt{1-\theta \rho^{2}+\eta_{t} \ln \rho^{2}}} \\
& =z_{0}+\frac{a}{\sqrt{2 N_{e} R_{i}}} \psi_{\theta}\left(\rho_{1}, \eta_{t}\right) .
\end{aligned}
$$

Let define formally the reversed to $\psi(\rho, \eta)$ function:

$$
x=\psi_{p}(\rho, \eta) \quad \rightarrow \quad \rho=\psi_{p}^{-1}(x, \eta) .
$$

Finally, after matching the trajectory within the bunch to that in the gap, we get the following conditions for the system to be periodic:

$$
\begin{aligned}
\frac{g}{2} & =\frac{a}{\sqrt{2 N_{i} R_{0}}}\left[\psi_{0}\left(\rho_{1}, \eta\right)-\psi_{0}\left(\rho_{m}, \eta\right)\right] ; \\
l_{t} & =\frac{a}{\sqrt{2 N_{e} R_{i}}} \psi_{1}\left(\rho_{1}, \eta\right) \\
\lambda & =\frac{2 a}{\sqrt{2 N_{e} R_{i}}} \psi_{1}\left(\rho_{*}, \eta\right) \\
2 l_{t} & =L_{t}-n \lambda ; \quad n=0,1,2, \ldots \\
\rho_{*} & =\exp \left[\frac{\rho_{1}^{2}-1}{2 \eta_{t}}\right] ; \\
\rho_{1} & =\psi_{1}^{-1}\left(\frac{l_{t}}{a} \sqrt{2 N_{e} R_{i}}, \eta_{t}\right)
\end{aligned}
$$

It can be seen that for the limiting case $\eta_{t} \rightarrow 0$ the stability conditions $[1,2]$ are deduced from (11).

\section{COMPUTATION OF THE CORE STABILITY LIMITS}

As it may be seen from the expressions obtained, the boundary lines of the stability islands can not be presented in an explicit analytical form. Then, a computer code was written for calculation of the limits of ion stability for both the case of round beam (considered above) and flat beam (derivation of the stability border for this case is similar to the round one). The reason is that for an actual beam of elliptic cross section, the stability bound must lay somewhere in between the flat and round ones.

Bunch intensity was presented with dimensionless parameter $\nu$ :

$$
\begin{aligned}
& \nu_{\text {round }}=\left(\frac{L_{t}}{a}\right)^{2} \frac{2 N_{i} R_{0}}{\pi^{2}} ; \\
& \nu_{\text {flat }}=\left(\frac{L_{t}}{a}\right)^{2} \frac{2 N_{i} R_{0}}{\pi} \frac{a}{b} .
\end{aligned}
$$

where $b$ is the width of a flat bunch.

The train neutralization factor $\eta_{t}$, and the relative bunch (train) length $g / L_{t}$ were the other two parameters.

Computed stability islands are presented in Figs 2-3 where numbers in line brakes show the relative gap length $g / L_{t}$. As is seen from the figures, stability limits are shifted toward higher beam densities with increase in the ion core density. Then, projections of the islands into the abscissae are wider then for $\eta=0$ and can overlap each other. Thus, the gap length shorter than some $g_{\mathrm{ol}}$ is not provided clearing.

\section{RESULTS}

The analytical and computational study on the selfconsistent stability of the ion core in the pulsed electron beam was carried out. As is yielded from the theory, relative core density reaches its maximum in the uniform, continuous beam. Any inhomogeneity in the longitudinal 


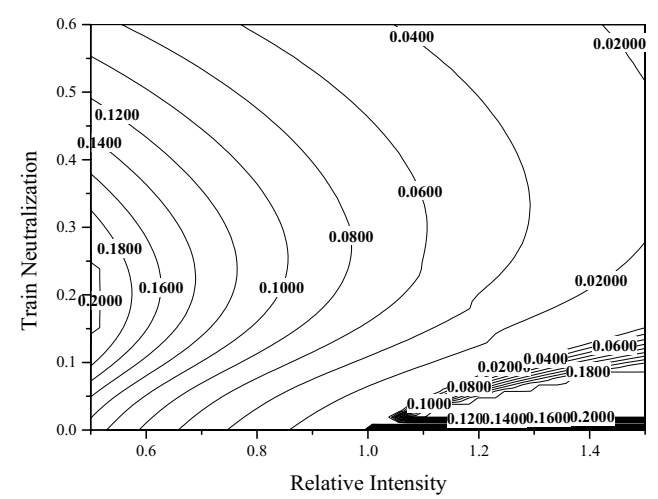

Figure 2: First stability islands; a cylinder beam.

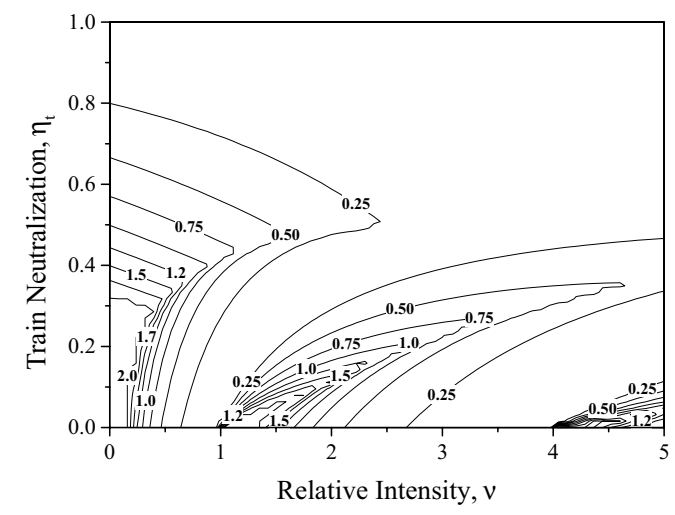

Figure 3: First stability islands; a flat beam.

beam density distribution is resulting in decreasing the core density. This fact is surprisingly opposite to expected from the theory of strong focusing where application of defocusing elements increases the transverse stability - repulsion of ions by the space charge field of the core causes decrease in the stability limit.

Maximal stable ion density decreases with increasing the bunch train density. Thus, the stable islands with big number (big number of ion oscillations per train, $K=[\sqrt{\nu}] \gg$ 1) are less harmful, for example, the island with $K=12$ and $g / L_{t}=2$ has the only $0.5 \%$ of neutralization in its maximum (see Fig 4).

Also it is worth to take into account that the space between the stable islands is increased with its number. Thus, the gap in bunch train is effective for removing of ions from the intense electron beams circulating in the storage ring with large circumference.

\section{REFERENCES}

[1] Y. Baconier, G. Brianty, "The Stability of Ions in Bunched Beam Machines", CERN/SPS/80-2 (DI) (1980)

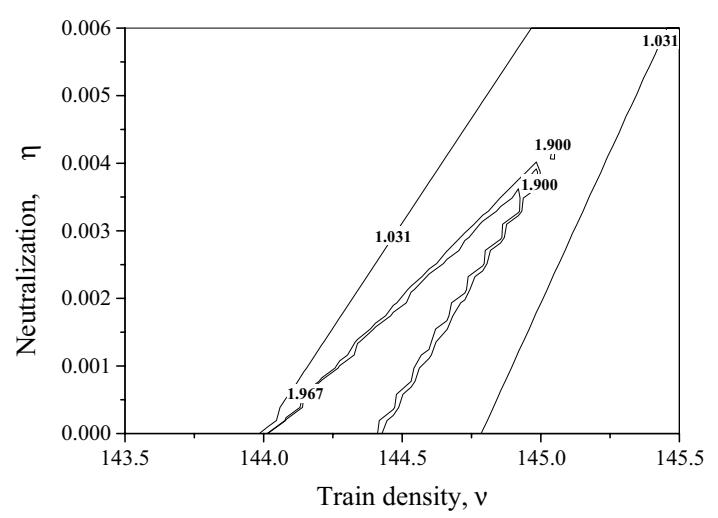

Figure 4: 12-th Stability island

[2] Y. Miyahara, "Parametric Resonance of Trapped Ions in Electron Storage Rings," NIM A366 p 221 (1995)

[3] E. Bulyak, "Capturing and Confinement of Ions by the Beam Circulating in an Electron Storage Ring", Sov. Physics JTF vol. 56, p. 72 (1986)

[4] E. Bulayk, "Ion Clearing Methods for the Electron Storage Rings”, Proc. EPAC-96 (Barcelona, Spain) p. 1078 (1996) 